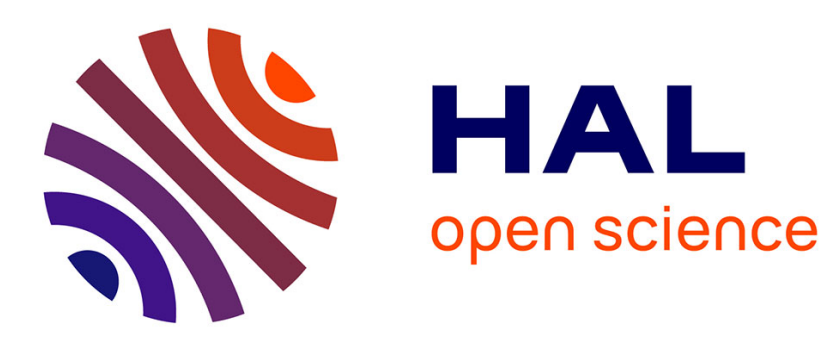

\title{
Slow admission and power control for small cell networks via distributed optimization
}

Siew Eng Nai, Tony Q.S. Quek, Mérouane Debbah

\section{To cite this version:}

Siew Eng Nai, Tony Q.S. Quek, Mérouane Debbah. Slow admission and power control for small cell networks via distributed optimization. WCNC 2013, Apr 2013, Shanghai, China. pp.2261 - 2265, 10.1109/WCNC.2013.6554912 . hal-00927760

\section{HAL Id: hal-00927760}

https://hal-centralesupelec.archives-ouvertes.fr/hal-00927760

Submitted on 13 Jan 2014

HAL is a multi-disciplinary open access archive for the deposit and dissemination of scientific research documents, whether they are published or not. The documents may come from teaching and research institutions in France or abroad, or from public or private research centers.
L'archive ouverte pluridisciplinaire HAL, est destinée au dépôt et à la diffusion de documents scientifiques de niveau recherche, publiés ou non, émanant des établissements d'enseignement et de recherche français ou étrangers, des laboratoires publics ou privés. 


\title{
Slow Admission and Power Control for Small Cell Networks via Distributed Optimization
}

\author{
Siew Eng Nai*, Tony Q. S. Quek*†, and Mérouane Debbah ${ }^{\ddagger}$ \\ *Institute for Infocomm Research, A*STAR, 1 Fusionopolis Way, \#21-01 Connexis, Singapore 138632 \\ †Singapore University of Technology and Design, 20 Dover Drive, Singapore 138682 \\ $\ddagger$ SUPELEC, 3 rue Joliot-Curie, 91192 Gif-sur-Yvette, France
}

\begin{abstract}
Although small cell networks are environmentally friendly and can potentially improve the coverage and capacity of cellular layers, it is imperative to control the interference in such networks before overlaying them in a macrocell network on a large-scale basis. In recent work, we developed the joint admission and power control algorithm for two-tier small cell networks in which the number of small cell users that can be admitted at their quality-of-service (QoS) constraints is maximized without violating the macrocell users' QoS constraints. The QoS metric adopted is outage probability. In this paper, we investigate the distributed implementation of the joint admission and power control problem where the small cells can determine jointly their admissibility and transmit powers autonomously.
\end{abstract}

\section{INTRODUCTION}

Energy consumption and electromagnetic pollution are fast becoming problems that future communication infrastructures need to alleviate. Towards this end, the design of green cellular networks has been considered. One such approach is to overlay a macrocell network with many small cells [1], [2]. With small cells, users can obtain better indoor reception and power savings due to the low transmit powers. In addition, small cells can offload much data traffic from the macrocell network via backhaul. This enhances the overall network coverage and capacity. However, one of the major impediments to the success of two-tier small cell networks is the presence of intertier and intra-tier interference.

A significant amount of research is focused on managing inter-tier and intra-tier interference [3]-[15]. In [3], inter-tier interference between the macrocell and small cell tiers can be avoided by using orthogonal spectrum allocation. Clearly, this method is inefficient given a sparse small cell deployment setting and a much higher area spectrum efficiency can be achieved by spectrum sharing [4]. On the other hand, for spectrum sharing in two-tier small cell networks, the intertier interference has to be properly controlled by using techniques such as access control [4]-[7], power control [8]-[10], multiple antennas [11], [12], or cognitive radio [13]-[15]. All these schemes [3]-[15] involve computational and signalling overhead. If the set of active small cells changes at the rate of Rayleigh fading, there will be very frequent updating and processing at the macrocell base station (MBS) and small cell access points (SAPs). Therefore, we proposed an interference management scheme with joint admission and power control that tracks at a much slower shadowing time-scale in [16].
In [16], we consider a two-tier small cell network, where small cell users (SUs) share the same spectrum with the macrocell user (MU). We assume that the MU has a higher spectrum access priority than the SUs. The proposed joint admission and power control problem aims to maximize the number of small cells admitted with their outage probability constraints satisfied and simultaneously, minimize their total transmit power, while guaranteeing the outage probability constraint of the MU. Different from conventional works [17], [18] which use instantaneous signal-to-interference-plusnoise ratio (SINR) constraints, we apply outage probability constraint for the users because this enables the admission and power updating to be performed at a much slower lognormal shadowing time-scale instead of the Rayleigh fading time-scale. As this formulation is NP-hard, convex relaxation is applied to obtain high quality approximate solutions that exhibit near optimal performance as shown in [16]. However, the centralized approach in [16] is difficult to implement in practice as the SAPs are randomly deployed by their subscribers.

In this paper, we investigate the decentralized implementation of the algorithm in [16] which is developed via dual decomposition. The distributed algorithm enables the small cells to self organize; the small cells can determine jointly (on their own) if they are admitted or rejected for communications and the respective transmit powers should they be admitted into the system. The simulation results show the effectiveness of our proposed distributed algorithm.

\section{System Model And Problem Formulation}

As shown in Fig. 1, we consider an uplink two-tier network where a macrocell network is overlaid with $N$ closed access small cells. The MBS and the SAPs are operating in a common frequency band with one $\mathrm{MU}^{1}$ and $N$ SUs. We assume that there is one $\mathrm{SU}$ in each small cell requesting to share the spectrum with the MU in order to communicate with its SAP. Therefore, the received SINR of the $i$ th SU can be written as

$$
\operatorname{SINR}_{i}^{\mathrm{s}}=\frac{G_{i i}^{\mathrm{ss}} F_{i i}^{\mathrm{ss}} P_{i}^{\mathrm{s}}}{\sum_{l=1, l \neq i}^{N} G_{i l}^{\mathrm{ss}} F_{i l}^{\mathrm{ss}} P_{l}^{\mathrm{s}}+G_{i 0}^{\mathrm{sm}} F_{i 0}^{\mathrm{sm}} P^{\mathrm{m}}+N_{o}}
$$

\footnotetext{
${ }^{1}$ A single MU is considered for brevity of exposition. More QoS constraints can be added to include multiple MUs which does not alter the structure of the proposed problem.
} 


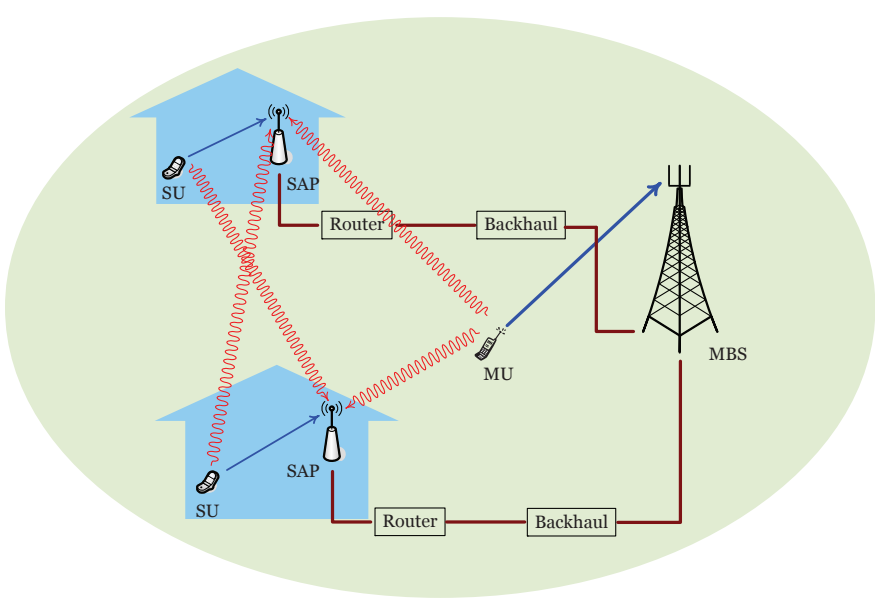

Fig. 1. Two-tier network where a macrocell network is overlaid with small cells. The straight arrows indicate the desired links while the wavy arrows indicates inter-tier and intra-tier interfering links.

where $G_{i l}^{\mathrm{ss}}$ and $F_{i l}^{\mathrm{ss}}$ denote the slow and fast fading gains from the $l$ th $\mathrm{SU}$ to the $i$ th SAP, respectively. In the following, we consider the slow fading gain to include the effect of propagation path loss and shadowing, and the fast fading gain is modeled as exponential power fading which corresponds to Rayleigh fading assumption. Similarly, $G_{i 0}^{\mathrm{sm}}$ and $F_{i 0}^{\mathrm{sm}}$ refers to the slow and fast fading gains from the MU (assigned index "0") to the $i$ th SAP, respectively. The transmit power of the $l$ th $\mathrm{SU}$ is $P_{l}^{\mathrm{s}}$, the transmit power of the $\mathrm{MU}$ is $P^{\mathrm{m}}$, which is assumed to be fixed as the MU does not cooperate with the SUs, and the noise power is $N_{o}$. With the Rayleigh fading assumption on the fast fading gains, $F_{i j}^{\mathrm{kl}}$ are independent exponentially distributed random variables with unit mean. Thus, the outage constraint of the $i$ th $\mathrm{SU}$ is given by $\operatorname{Pr}\left(\operatorname{SINR}_{i}^{\mathrm{s}} \leq \gamma_{i}^{\mathrm{s}}{ }^{\text {th }}\right) \leq \rho_{i}^{\mathrm{s}}$,th and derived as [10]

$$
\begin{aligned}
& \frac{b_{i}^{\mathrm{s}} N_{o}}{P_{i}^{\mathrm{s}}}+\sum_{l=1, l \neq i}^{N} \ln \left(1+\frac{b_{i}^{\mathrm{s}} G_{i l}^{\mathrm{ss}} P_{l}^{\mathrm{s}}}{P_{i}^{\mathrm{s}}}\right) \\
& +\ln \left(1+\frac{b_{i}^{\mathrm{s}} G_{i 0}^{\mathrm{sm}} P^{\mathrm{m}}}{P_{i}^{\mathrm{s}}}\right) \leq-\ln \left(1-\rho_{i}^{\mathrm{s}, \mathrm{th}}\right)
\end{aligned}
$$

where $\gamma_{i}^{\text {s,th }}$ and $\rho_{i}^{\text {s,th }}$ denote the pre-specified SINR and outage probability thresholds of the $i$ th SU, respectively, and for notational convenience, $b_{i}^{\mathrm{s}} \triangleq \gamma_{i}^{\mathrm{s}, \mathrm{th}} / G_{i i}^{\mathrm{ss}}$.

When SUs are operating, the received SINR of the MU is

$$
\operatorname{SINR}^{\mathrm{m}}=\frac{G_{\mathrm{M} 0}^{\mathrm{mm}} F_{\mathrm{M} 0}^{\mathrm{mm}} P^{\mathrm{m}}}{\sum_{i=1}^{N} G_{\mathrm{M} i}^{\mathrm{ms}} F_{\mathrm{M} i}^{\mathrm{ms}} P_{i}^{\mathrm{s}}+N_{o}}
$$

where $G_{\mathrm{M} 0}^{\mathrm{mm}}$ and $F_{\mathrm{M} 0}^{\mathrm{mm}}$ are the slow and fast fading gains from the MU to the MBS and $G_{\mathrm{M} i}^{\mathrm{ms}}$ and $F_{\mathrm{M} i}^{\mathrm{ms}}$ are the slow and fast fading gains from the $i$ th SU to the MBS. The outage constraint of the MU is then given by $\operatorname{Pr}\left(\operatorname{SINR}^{\mathrm{m}} \leq \gamma^{\mathrm{m}, \mathrm{th}}\right) \leq$ $\rho^{\mathrm{m}, \mathrm{th}}$ and derived as

$$
\sum_{i=1}^{N} \ln \left(1+b_{\mathrm{M} i}^{\mathrm{ms}} P_{i}^{\mathrm{s}}\right) \leq \ln \mu^{\mathrm{m}}
$$

where $\rho^{\mathrm{m}, \mathrm{th}}$ is the pre-specified outage probability threshold of the MU, $\mu^{\mathrm{m}}=\left(1-\bar{\rho}^{\mathrm{m}}\right) /\left(1-\rho^{\mathrm{m}, \mathrm{th}}\right), \bar{\rho}^{\mathrm{m}}$ is the outage probability of the MU in the absence of SUs, and for notational convenience, $b_{\mathrm{M} i}^{\mathrm{ms}} \triangleq\left(\gamma^{\mathrm{m}, \mathrm{th}} G_{\mathrm{M} i}^{\mathrm{ms}}\right) /\left(G_{\mathrm{M} 0}^{\mathrm{mm}} P^{\mathrm{m}}\right)$.

The objective of this work is to maximize the number of SUs that can be admitted with a guaranteed QoS while guaranteeing the QoS of the MU and simultaneously minimize the total transmission power of the SUs. In this paper, the QoS provided to the MU as well as the SUs is outage probability constraint. Following the approach in [17], we can provide a compact and elegant single-stage framework [16] which is given by

$$
\begin{array}{ccc}
\min _{P_{i}^{\mathrm{s}}, s_{i}} & \epsilon \sum_{i=1}^{N} P_{i}^{\mathrm{s}}+(1-\epsilon) \sum_{i=1}^{N} \frac{1}{s_{i}+1} & \\
\text { s.t. } & 0 \leq P_{i}^{\mathrm{s}} \leq P^{\mathrm{s}, \max }, & \forall i \\
& s_{i} \in[0,1], & \forall i \\
& \frac{s_{i} b_{i}^{\mathrm{s}} N_{o}}{P_{i}^{\mathrm{s}}}+\sum_{l=1, l \neq i}^{N} \ln \left(1+\frac{s_{i} b_{i}^{\mathrm{s}} G_{i l}^{\mathrm{ss}} P_{l}^{\mathrm{s}}}{P_{i}^{\mathrm{s}}}\right) & \\
+\ln \left(1+\frac{s_{i} b_{i}^{\mathrm{s}} G_{i 0}^{\mathrm{sm}} P^{\mathrm{m}}}{P_{i}^{\mathrm{s}}}\right) \leq-\ln \left(1-\rho_{i}^{\mathrm{s}}, \mathrm{th}\right. & , \forall i \\
& \sum_{i=1}^{N} \ln \left(1+b_{\mathrm{M} i}^{\mathrm{ms}} P_{i}^{\mathrm{s}}\right) \leq \ln \mu^{\mathrm{m}} &
\end{array}
$$

where the auxiliary scheduling variables are $s_{i} \in[0,1], P^{\mathrm{s}, \max }$ is the maximum transmit power of each $\mathrm{SU}$, the value of $s_{i}$ determines the admissibility of the $i$ th $\mathrm{SU}$ and if the outage constraint of the $i$ th SU is taken into consideration in the power control part of the joint admission and power control problem. If $s_{i}=0$, the $i$ th $\mathrm{SU}$ is rejected and (5d) reduces to the trivial inequality $\ln \left(1-\rho_{i}^{\mathrm{s}, \text { th }}\right) \leq 0$; if $s_{i}=1$, the $i$ th $\mathrm{SU}$ is scheduled for admission and ( $5 \mathrm{~d}$ ) becomes an active constraint. The cost function consists of the weighted sum of transmit powers of the SUs which is bounded and the admission cost which is discrete-valued. Intuitively, the weighing parameter $\epsilon<\epsilon^{*}$ has to be small enough in order to ensure that admission control is always prioritized before power control. The choice of $\epsilon$ can be understood by the following interpretation that dropping any user costs more than can possibly be saved by total power minimization [17].

Remark 1: By choosing $\epsilon<\epsilon^{*}=1 /\left(2 N P^{\mathrm{s}, \max }+1\right)$, the solution of (5) is equivalent to firstly, finding the optimal set $\tilde{\mathcal{S}}$ that contains the largest number of SUs that can be admitted subject to transmit power constraints $(5 \mathrm{~b})$, outage probability constraints of the SUs (2), and outage probability constraint of the MU (4) and lastly, minimizing the total transmit power of the SUs in $\tilde{\mathcal{S}}$ subject to the same constraints (5b), (2), and (4).

Proof: The proof is omitted for brevity.

Different from [17] which constrains the instantaneous SINRs of the SUs, the proposed formulation constrains the outage probabilities of the MU and SUs. Consequently, the objective function, weighing parameter $\epsilon$, and the effect that the scheduling variables $s_{i}$ exert on the constraints in (5) are entirely different from those in [17].

\section{CONVEX RELAXATION}

The single-stage reformulation in (5) is non-convex due to the binary constraints $(5 \mathrm{c})$ and the term $1 /\left(s_{i}+1\right)$ in the 
objective function being neither a posynomial nor a monomial. Therefore, we apply the technique of convex relaxation. First, we relax the binary constraints to allow $s_{i}$ to take on any real value within the interval $[0,1]$. Next, we approximate $f\left(s_{i}\right)=1 /\left(s_{i}+1\right)$ with a monomial, i.e., $c s_{i}^{\alpha}=0.5 s_{i}^{-\frac{1}{2}}$ (details are skipped for brevity) such that the entire optimization problem can be cast as a geometric programming (GP) problem. Finally, the new convex single-stage formulation is obtained as follows:

$$
\begin{array}{cc}
\min _{P_{i}^{\mathrm{s}}, s_{i}} \epsilon \sum_{i=1}^{N} P_{i}^{\mathrm{s}}+c(1-\epsilon) \sum_{i=1}^{N} s_{i}^{-\alpha} & \\
\text { s.t. } & 0 \leq s_{i} \leq 1, \\
& (5 b),(5 d),(5 e)
\end{array} \quad \forall i
$$

which is clearly a GP and it can be solved globally and efficiently. After (6) is solved, if all $s_{i}=1$, it means that all the MU and SUs can be served while satisfying their outage constraints. Otherwise, the removal of SUs is triggered in order to admit the maximum number of SUs with their outage constraints and that of the MU met. In this paper, the iterative removal algorithm is used to remove the SU with the minimal $s_{i \mid s_{i} \neq 1}$ (one by one) at each iteration of (6). The algorithm terminates when $s_{i}=1$ for all the remaining SUs.

\section{Distributed Optimization}

Although (6) can be efficiently solved in polynomial time, it still requires a centralized network controller to solve the optimization problem. Due to the random deployment of the small cells, distributed algorithms are especially favoured so that the small cells can determine their own admissibility and transmit powers (if they are admitted for communications). To this end, the dual decomposition approach is applied [19]. As (5d) is coupled with the transmit powers of other SUs, an auxiliary variable $z_{i l}=G_{i l}^{\mathrm{ss}} P_{l}^{\mathrm{s}}$ and an additional equality (7d) are introduced. Let $\tilde{s}_{i}=\ln s_{i}, \tilde{P}_{i}^{\mathrm{s}}=\ln P_{i}^{\mathrm{s}}$, and $\tilde{z}_{i l}=\ln z_{i l}$, then we can rewrite (6) as follows:

$$
\begin{array}{cc}
\min _{\tilde{P}_{i}^{\mathrm{s}}, \tilde{s}_{i}} \epsilon \sum_{i=1}^{N} \exp \left(\tilde{P}_{i}^{\mathrm{s}}\right)+c(1-\epsilon) \sum_{i=1}^{N}\left(\exp \left(\tilde{s}_{i}\right)\right)^{-\alpha} & \text { (7a) } \\
\text { s.t. } & \tilde{P}_{i}^{\mathrm{s}} \leq \ln P^{\mathrm{s}, \max }, \quad \forall i
\end{array}
$$

Let $\gamma_{i l}$ be the consistency prices corresponding to (7d), $\zeta_{i}$ and $\lambda$ be the dual variables for (7e) and (7f), respectively so the partial Lagrangian for (7) can be written as

$$
\begin{aligned}
L\left(\tilde{P}_{i}^{\mathrm{s}}, \tilde{s}_{i}, \tilde{z}_{i l}\right) & =\epsilon \sum_{i=1}^{N} \exp \left(\tilde{P}_{i}^{\mathrm{s}}\right)+c(1-\epsilon) \sum_{i=1}^{N}\left(\exp \left(\tilde{s}_{i}\right)\right)^{-\alpha} \\
& +\sum_{i=1}^{N} \zeta_{i}\left[\frac{\exp \left(\tilde{s}_{i}\right) b_{i}^{\mathrm{s}} N_{o}}{\exp \left(\tilde{P}_{i}^{\mathrm{s}}\right)}\right. \\
& +\sum_{l=1, l \neq i}^{N} \ln \left(1+\frac{\exp \left(\tilde{s}_{i}\right) b_{i}^{\mathrm{s}} \exp \left(\tilde{z}_{i l}\right)}{\exp \left(\tilde{P}_{i}^{\mathrm{s}}\right)}\right) \\
& \left.+\ln \left(1+\frac{\exp \left(\tilde{s}_{i}\right) b_{i}^{\mathrm{s}} G_{i 0}^{\mathrm{sm}} P^{\mathrm{m}}}{\exp \left(\tilde{P}_{i}^{\mathrm{s}}\right)}\right)+\ln \left(1-\rho_{i}^{\mathrm{s}, \mathrm{th}}\right)\right] \\
& +\lambda\left[\sum_{i=1}^{N} \ln \left(1+b_{M i}^{\mathrm{ms}} \exp \left(\tilde{P}_{i}^{\mathrm{s}}\right)\right)-\ln \mu^{\mathrm{m}}\right] \\
& +\sum_{i=1}^{N} \sum_{l=1, l \neq i}^{N} \gamma_{i l}\left[\tilde{z}_{i l}-\ln G_{i l}^{\mathrm{ss}}-\tilde{P}_{l}^{\mathrm{s}}\right]
\end{aligned}
$$

where for each $\mathrm{SU}, i=1, \cdots, N$, we have

$$
\begin{aligned}
L_{i}\left(\tilde{P}_{i}^{\mathrm{s}}, \tilde{s}_{i}, \tilde{z}_{i l}\right) & =\epsilon \exp \left(\tilde{P}_{i}^{\mathrm{s}}\right)+c(1-\epsilon)\left(\exp \left(\tilde{s}_{i}\right)\right)^{-\alpha} \\
& +\zeta_{i}\left[\frac{\exp \left(\tilde{s}_{i}\right) b_{i}^{\mathrm{s}} N_{o}}{\exp \left(\tilde{P}_{i}^{\mathrm{s}}\right)}\right. \\
& +\sum_{l=1, l \neq i}^{N} \ln \left(1+\frac{\exp \left(\tilde{s}_{i}\right) b_{i}^{\mathrm{s}} \exp \left(\tilde{z}_{i l}\right)}{\exp \left(\tilde{P}_{i}^{\mathrm{s}}\right)}\right) \\
& \left.+\ln \left(1+\frac{\exp \left(\tilde{s}_{i}\right) b_{i}^{\mathrm{s}} G_{i 0}^{\mathrm{sm}} P^{\mathrm{m}}}{\exp \left(\tilde{P}_{i}^{\mathrm{s}}\right)}\right)\right] \\
& +\lambda \ln \left(1+b_{M i}^{\mathrm{ms}} \exp \left(\tilde{P}_{i}^{\mathrm{s}}\right)\right) \\
& +\sum_{l=1, l \neq i}^{N} \gamma_{i l} \tilde{z}_{i l}-\left(\sum_{l=1, l \neq i}^{N} \gamma_{l i}\right) \tilde{P}_{i}^{\mathrm{s}} .
\end{aligned}
$$

The consistency prices $\gamma_{i l}$ and Lagrange multipliers $\zeta_{i}$ and $\lambda$ can be updated according to the following equations:

$$
\begin{aligned}
\gamma_{i l}(t+1)= & \gamma_{i l}(t)+\delta_{\gamma}(t)\left[\tilde{z}_{i l}-\ln G_{i l}^{\mathrm{ss}}-\tilde{P}_{l}^{\mathrm{s}}\right], \\
\zeta_{i}(t+1)= & \zeta_{i}(t)+\delta_{\zeta}(t)\left[\frac{\exp \left(\tilde{s}_{i}\right) b_{i}^{\mathrm{s}} N_{o}}{\exp \left(\tilde{P}_{i}^{\mathrm{s}}\right)}\right. \\
+ & \sum_{l=1, l \neq i}^{N} \ln \left(1+\frac{\exp \left(\tilde{s}_{i}\right) b_{i}^{\mathrm{s}} \exp \left(\tilde{z}_{i l}\right)}{\exp \left(\tilde{P}_{i}^{\mathrm{s}}\right)}\right) \\
& \left.+\ln \left(1+\frac{\exp \left(\tilde{s}_{i}\right) b_{i}^{\mathrm{s}} G_{i 0}^{\mathrm{sm}} P^{\mathrm{m}}}{\exp \left(\tilde{P}_{i}^{\mathrm{s}}\right)}\right)+\ln \left(1-\rho_{i}^{\mathrm{s}, \mathrm{th}}\right)\right], \\
\lambda(t+1)= & \lambda(t)+\delta_{\lambda}(t)\left[\sum_{i=1}^{N} \ln \left(1+b_{M i}^{\mathrm{ms}} \exp \left(\tilde{P}_{i}^{\mathrm{s}}\right)\right)-\ln \mu^{\mathrm{m}}\right]
\end{aligned}
$$

where $t$ is the iteration index and $\delta_{\gamma}, \delta_{\zeta}$, and $\delta_{\lambda}$ are step sizes, respectively.

Incorporating all the above equations, we summarize our proposed distributed joint admission and power control algorithm as follows: 
1) At $t=0$, initialize $\gamma_{i l}=0$ for $\forall i, l, \lambda>0, \zeta_{i}>0$, and $\tilde{z}_{i l}=0$. First, each SAP $i$ estimates the interference from the MU, i.e., $G_{i 0}^{\mathrm{sm}} \mathrm{P}^{\mathrm{m}}$, and channel gains from other SUs, i.e., $G_{i l}^{\mathrm{ss}}$. Each SAP $i$ can get $\gamma^{\mathrm{m}, \mathrm{th}}, G_{M 0}^{\mathrm{mm}}, P^{\mathrm{m}}$, and $\mu^{\mathrm{m}}$ from the MBS using the backhaul. Each SU $i$ can obtain $G_{M i}^{\mathrm{ms}}$ via estimating $G_{i M}^{\mathrm{sm}}$ when the MBS is broadcasting or transmitting, after which it informs its SAP.

2) At $t \geq 1$, each $\mathrm{SAP} i$ solves (9) subject to its transmit power constraint $(7 \mathrm{~b})$ for $\tilde{P}_{i}^{\mathrm{s}}$ and $\tilde{s}_{i}$ using interior point method.

3) Each SAP $i$ checks if $\tilde{P}_{i}^{\mathrm{s}}$ and $\tilde{s}_{i}$ have converged. If so, go to Step (5). Otherwise, the SAP $i$ broadcasts its $\tilde{P}_{i}^{\mathrm{s}}$ or passes this information to other SAPs over a low-rate control channel.

4) The SAPs can appoint one of themselves as an acting SAP controller to pass their $G_{M i}^{\mathrm{ms}}$ to. The acting SAP controller updates $\lambda$ and informs the other SAPs of $\lambda$ via the low-rate control channel. Each SAP $i$ updates $\tilde{z}_{i l}, \gamma_{i l}$, and $\zeta_{i}$. Go to Step (2).

5) After $\tilde{P}_{i}^{\mathrm{s}}$ and $\tilde{s}_{i}$ have converged, each SAP can calculate $P_{i}^{s}$ and $s_{i}$. If $s_{i}=1$, the SU $i$ is admitted to share the spectrum with the MU and the SU $i$ then transmits at $P_{i}^{\mathrm{s}}$ to communicate with the respective SAP. If $s_{i}=0$, the SU $i$ is rejected and it does not transmit at all.

\section{Simulation Results}

The performance of our proposed distributed joint admission and power control algorithm is investigated for a code division multiple access system. The MBS is located in the centre of a square area of length $2000 \mathrm{~m}$. The small cells are randomly located in the same area excluding a square area of length $100 \mathrm{~m}$ centred at the MBS. The SAP is located at the centre of each small cell (square area) and the SU is randomly located at either one of the four corners of the cell at a distance of $20 \mathrm{~m}$. The small cells are separated from each other by at least $1 \mathrm{~m}$. The MU is randomly located outside the small cells by at least $1 \mathrm{~m}$. The noise power at the MBS and SAPs is $N_{o}=10^{-10} \mathrm{~W}$. The transmit power and SINR threshold of the $\mathrm{MU}$ are $P^{\mathrm{m}}=4 \mathrm{~W}$ and $\gamma^{\mathrm{m} \text {,th }}=0 \mathrm{~dB}$, respectively. The total number of requesting SUs is 3 . The SINR thresholds of the SUs is $25 \mathrm{~dB}$. The maximum transmit power of the SUs is $P^{\mathrm{s}, \max }=1 \mathrm{~W}$. The processing gains of the MBS and SAPs are $P G^{\mathrm{m}}=10$ and $P G_{i}^{\mathrm{s}}=1$, respectively. The $\mathrm{MU}$ and SUs have an outage probability threshold $\rho^{\mathrm{m} \text {,th }}=10 \%$ and $\rho_{i}^{\mathrm{s}, \text { th }}=10 \%$. The slow fading gain between transmitter $j$ and receiver $i$ is modeled as $G_{i j}=K_{0} \times 10^{\beta_{i j} / 10} \times d_{i j}^{-\eta}$ where $d_{i j}$ is the distance between them, $K_{0}=10^{3}$ is a factor to include the effects of antenna gain and carrier frequency, $\beta_{i j}$ is a Gaussian random variable with zero mean and standard deviation of $4 \mathrm{~dB}$ to account for log-normal shadowing effects, and the path loss exponent is $\eta=3$ except that $\eta=4$ for $G_{i i}^{s s}$.

We study the performance of the proposed distributed joint admission and power control algorithm by comparing with its centralized counterpart. In the first example in Fig. 2, the centralized algorithm admits all the requesting SUs while in the second example in Fig. 3, the centralized algorithm admits

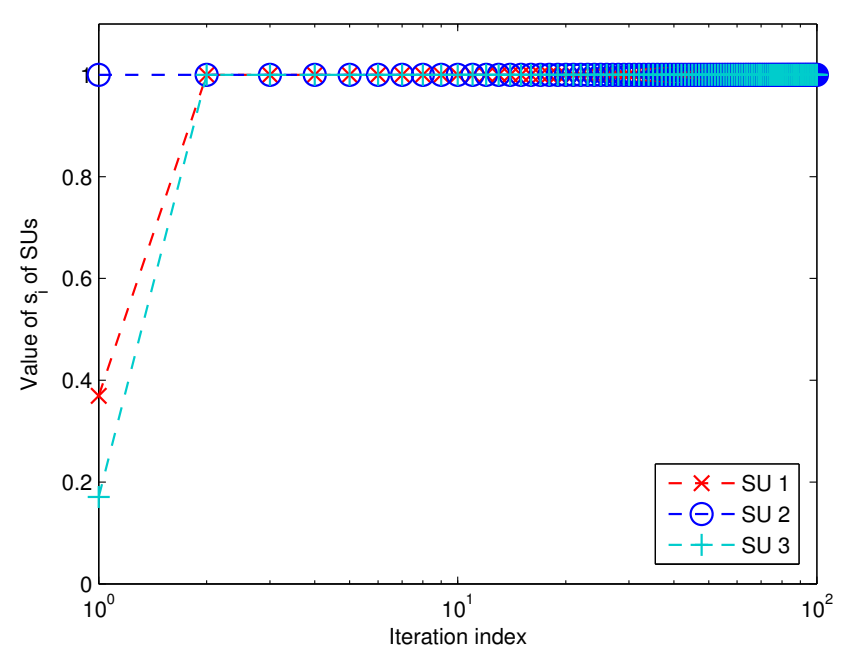

(a)

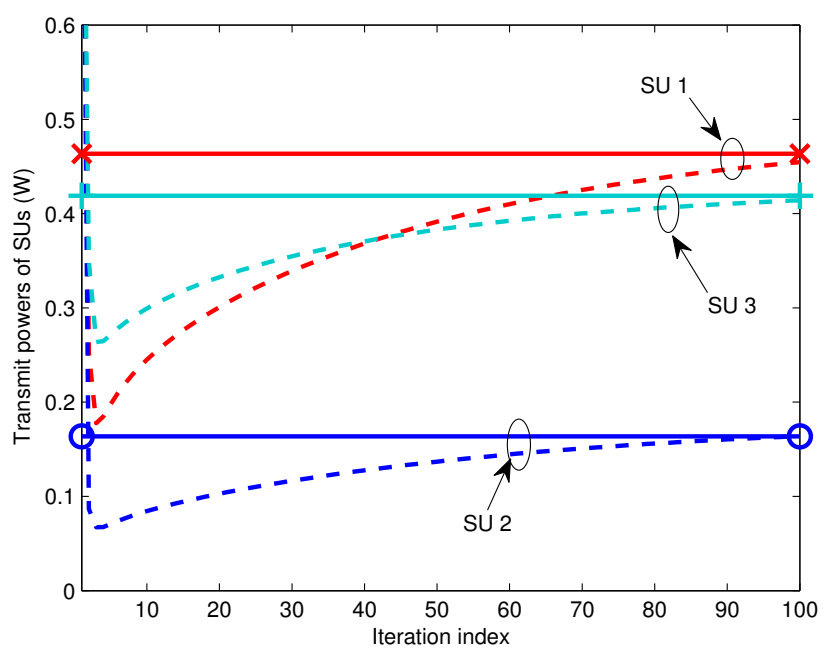

(b)

Fig. 2. (a) Value of $s_{i}$ of SUs obtained by the proposed distributed algorithm versus iteration index. All SUs are admitted. Note that the Xaxis is on a logarithm scale. (b) Transmit powers of SUs obtained by the proposed distributed algorithm versus iteration index. The solid lines indicate the transmit powers of the respective SUs as obtained by the centralized algorithm.

only 2 SUs which are SU 2 and SU 3. Fig. 2(a) and Fig. 3(a) show that the distributed algorithm admits the same SUs as that of the centralized algorithm. Note that the x-axis of Fig. 2(a) is on a logarithm scale. Fig. 2(b) and Fig. 3(b) show that the transmit powers of the admitted SUs converge to those obtained by the centralized algorithm (as indicated by the solid lines). In Fig. 3(b), the transmit power of SU 1 after the convergence of the distributed algorithm is not important because it is not admitted for communications since $s_{1} \neq 1$ in Fig. 3(a), hence SU 1 will not transmit at all. 


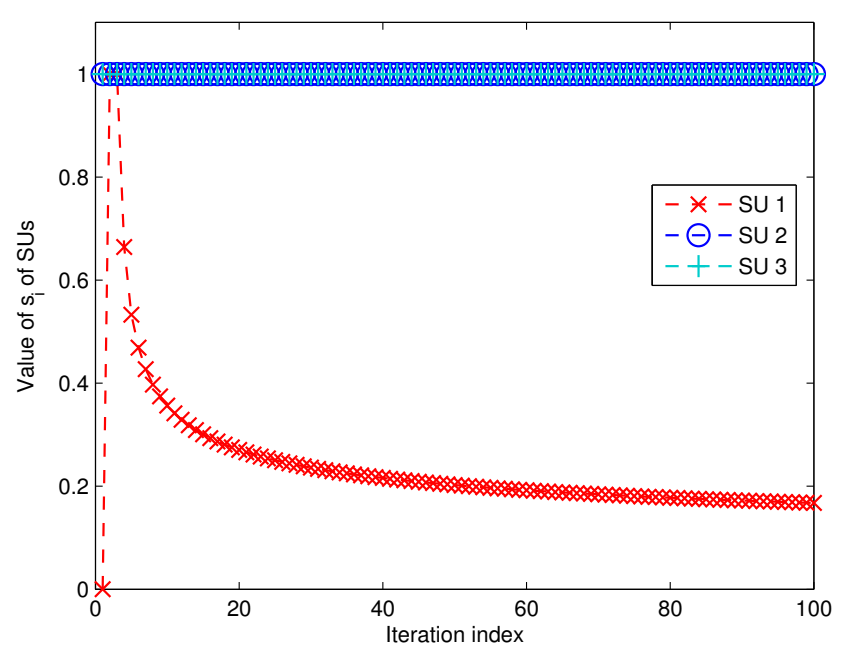

(a)

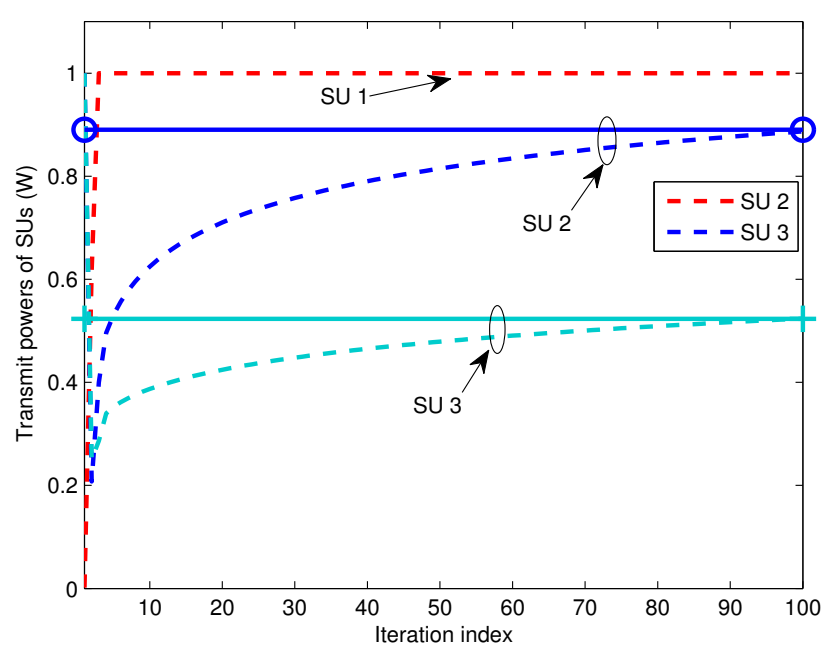

(b)

Fig. 3. (a) Value of $s_{i}$ of SUs obtained by the proposed distributed algorithm versus iteration index. Only SU 2 and SU 3 are admitted. (b) Transmit powers of SUs obtained by the proposed distributed algorithm versus iteration index. The solid lines indicate the transmit powers of the respective SUs as obtained by the centralized algorithm.

\section{CONCLUSION}

In this paper, we investigated the distributed implementation of a shadowing time-scale based joint admission and power control algorithm in two-tier small cell networks. In particular, the number of small cell users that can be admitted at their outage probability specifications is maximized and their total transmit power is minimized while guaranteeing the outage probability specification of the macrocell user. As this joint admission and power control problem is NP-hard, convex relaxation is applied to obtain high quality approximate solutions. The distributed implementation is developed by applying dual decomposition technique which empowers the small cells to determine their own admissibility into the system and the transmit powers if they are admitted.

\section{ACKNOWLEGEMENT}

This work was supported by the SRG ISTD 2012037 and the CAS Fellowships for Young International Scientists.

\section{REFERENCES}

[1] J. Hoydis, M. Kobayashi, and M. Debbah, "Green small-cell networks," IEEE Veh. Technol. Mag., vol. 6, no. 1, pp. 37-43, Mar. 2011.

[2] D. López-Pérez, İ. Güvenc, G. de la Roche, M. Kountouris, T. Q. S. Quek, and J. Zhang, "Enhanced intercell interference coordination challenges in heterogeneous networks," IEEE Wireless Commun., vol. 18, no. 3, pp. 22-30, Jun. 2011.

[3] V. Chandrasekhar and J. G. Andrews, "Spectrum allocation in tiered cellular networks," IEEE Trans. Commun., vol. 57, no. 10, pp. 30593068, Oct. 2009.

[4] W. C. Cheung, T. Q. S. Quek, and M. Kountouris, "Throughput optimization, spectrum allocation, and access control in two-tier femtocell networks," IEEE J. Sel. Areas Commun., vol. 30, no. 3, pp. 561-574, Apr. 2012.

[5] A.-H. Tsai, L.-C. Wang, J.-H. Huang, and R.-B. Hwang, "High-capacity OFDMA femtocells by directional antennas and location awareness," IEEE Syst. J., vol. 6, no. 2, pp. 329-340, Jun. 2012.

[6] C.-H. Ko and H.-Y. Wei, "On-demand resource-sharing mechanism design in two-tier OFDMA femtocell networks," IEEE Trans. Veh. Technol., vol. 60, no. 3, pp. 1059-1071, Mar. 2011.

[7] P. Xia, V. Chandrasekhar, and J. G. Andrews, "Open vs. closed acess femtocells in the uplink," IEEE Trans. Wireless Commun., vol. 9, no. 12, pp. 3798-3809, Dec. 2010.

[8] V. Chandrasekhar, J. G. Andrews, T. Muharemovic, Z. Shen, and A. Gatherer, "Power control in two-tier femtocell networks," IEEE Trans. Wireless Commun., vol. 8, no. 8, pp. 4316-4328, Aug. 2009.

[9] C. W. Tan, S. Friedland, and S. H. Low, "Spectrum management in multiuser cognitive wireless networks: Optimality and algorithm," IEEE J. Sel. Areas Commun., vol. 29, no. 2, pp. 421-430, Feb. 2011.

[10] C. W. Tan, "Optimal power control in Rayleigh-fading heterogeneous networks," in Proc. IEEE Int. Conf. Comput. Commun., Shanghai, China, Apr. 2011, pp. 2552-2560.

[11] S. Park, W. Seo, Y. Kim, S. Lim, and D. Hong, "Beam subset selection strategy for interference reduction in two-tier femtocell networks," IEEE Trans. Wireless Commun., vol. 9, no. 11, pp. 3440-3449, Nov. 2010.

[12] Y. Jeong, T. Q. S. Quek, and H. Shin, "Beamforming optimization for multiuser two-tier networks," J. Commun. Netw., vol. 13, no. 4, pp. 327 338, Aug. 2011.

[13] S.-M. Cheng, S.-Y. Lien, F.-S. Chu, and K.-C. Chen, "On exploiting cognitive radio to mitigate interference in macro/femto heterogeneous networks," IEEE Wireless Commun., vol. 18, no. 3, pp. 40-47, Jun. 2011.

[14] S.-Y. Lien, Y.-Y. Lin, and K.-C. Chen, "Cognitive and game-theoretical radio resource management for autonomous femtocells with QoS guarantees," IEEE Trans. Wireless Commun., vol. 10, no. 7, pp. 2196-2206, Jul. 2011.

[15] A. Adhikary, V. Ntranos, and G. Caire, "Cognitive femtocells: Breaking the spatial reuse barrier of cellular systems," in Proc. Inform. Theory Appl. Workshop, San Diego, CA, Feb. 2011, pp. 1-10.

[16] S. E. Nai, T. Q. S. Quek, and M. Debbah, "Shadowing time-scale admission and power control for small cell networks," in Proc. IEEE Int. Symp. Wireless Pers. Multimedia Commun., Taipei, Taiwan, Sep. 2012.

[17] E. Matskani, N. D. Sidiropoulos, Z.-Q. Luo, and L. Tassiulas, "Convex approximation techniques for joint multiuser downlink beamforming and admission control," IEEE Trans. Wireless Commun., vol. 7, no. 7, pp. 2682-2693, Jul. 2008.

[18] I. Mitliagkas, N. D. Sidiropoulos, and A. Swami, "Joint power and admission control for ad-hoc and cognitive underlay networks: Convex approximation and distributed implementation," IEEE Trans. Wireless Commun., vol. 10, no. 12, pp. 4110-4121, Dec. 2011.

[19] M. Chiang, Geometric Programming for Communication Systems. Hanover, MA: Now Publishers Inc., 2005. 